\title{
Gestational Diabetes Mellitus, A2
}

National Cancer Institute

\section{Source}

National Cancer Institute. Gestational Diabetes Mellitus, A2. NCI Thesaurus. Code

C112845.

Carbohydrate intolerance first diagnosed during pregnancy. Diagnosis from abnormal oral glucose tolerance test (OGTT) and abnormal fasting or post-prandial glucose levels. Euglycemia achieved with medication. 